\title{
Hydrothermal production and gel filtration purification of xylo-oligosaccharides from rice straw
}

\author{
Patrícia Moniz $^{\mathrm{a}, \mathrm{b}}$, Helena Pereira ${ }^{\mathrm{b}}$, Luís C. Duarte ${ }^{\mathrm{a}}$, Florbela Carvalheiro ${ }^{\mathrm{a}, *}$ \\ a Unidade de Bioenergia, LNEG - Laboratório Nacional de Energia e Geologia, Estrada do Paço do Lumiar, 22, Lisboa 1649-038, Portugal \\ ${ }^{\mathrm{b}}$ Centro de Estudos Florestais, Instituto Superior de Agronomia, Universidade de Lisboa, 1349-017 Lisboa, Portugal
}

\section{A R T I C L E I N F O}

\section{Article history:}

Received 19 June 2014

Received in revised form 4 September 2014

Accepted 9 September 2014

Available online 28 September 2014

\section{Keywords:}

Agricultural residues

Autohydrolysis

Gel filtration chromatography

Hemicellulose

Xylan

\begin{abstract}
A B S T R A C T
Hydrothermal treatment (autohydrolysis) is an advantageous alternative to fractionate biomass that was not yet explored for rice straw. In this work, the process was optimised and proved to be highly selective towards hemicellulose. Hydrolysates containing a mixture of oligomeric compounds (mainly xylo-oligosaccharides, XOS), could be obtained under relatively mild operation conditions $\left(210^{\circ} \mathrm{C}\right.$, $\log R_{0}=3.59$ ), yielding a maximum of $40.1 \mathrm{~g} / 100 \mathrm{~g}$ of initial xylan. The produced XOS were separated by molecular mass using gel filtration chromatography (GFC). Different fractions of purified XOS were obtained ranging from small polysaccharides and high DP oligosaccharides ( $D P \geq 23$ ), to medium and low DP oligosaccharides ( $\mathrm{DP} \geq 3$ ), and separated fractions of by-products (acetic acid, furan derivatives and phenols) as well as di-, and monosaccharides. GFC was an efficient purification method enabling the recovery of interesting categories of XOS that can have potential applications to the pharma, food and feed industries.
\end{abstract}

(C) 2014 Elsevier B.V. All rights reserved.

\section{Introduction}

Agricultural residues and by-products have gained increased attention as potential substrates for the production of fuels, chemicals and bio-materials (Kamm and Kamm, 2007).

Rice is one of the major grain crops worldwide and its residues (straw and husks) are produced in large quantities. Rice straw is usually considered a residue or even a waste material although it may be used as mulching material, feed, compost substrate, cattle house flooring, as well as for straw handicraft and combustion (Kadam et al., 2000; Matsumura et al., 2005). But, conversely to other similar materials, the use of rice straw for feeding or for combustion for energy production may cause problems due to its high silica content (Domínguez-Escribá and Porcar, 2009) and thus most of this material is left unused in the fields.

An alternative for rice straw valorisation can be the production of oligosaccharides within the biorefinery framework along with the production of biofuels. Rice wastes can be a major single feedstock for bioethanol production as both straw and husks have high polysaccharide and low lignin contents, with the straw presenting higher hemicellulose content (Binod et al., 2010;

\footnotetext{
* Corresponding author. Tel.: +35121 0924713; fax: +351217163636.

E-mail address: florbela.carvalheiro@lneg.pt (F. Carvalheiro).
}

Domínguez-Escribá and Porcar, 2009). Prior to bioethanol production, several pretreatments have to be applied to rice straw in order to increase its upgradability. Previously studied pretreatments mainly include dilute acid, alkali (including ammonia) and enzymatic methods (Binod et al., 2010). However such pretreatments are expensive and/or time-consuming, not selective, require neutralisation, or result in the formation of by-products that can inhibit subsequent fermentation. Furthermore, none of these methods is suitable for oligosaccharides production from biomass, as either they degrade oligosaccharides (dilute acid hydrolysis), are not selective (alkaline treatments), or present very low yields (enzymatic processes).

An alternative option for the valorisation of rice straw under the biorefinery approach is the hydrolysis of hemicellulose by hydrothermal processing (autohydrolysis), which is an effective pretreatment enabling a high recovery of soluble saccharides in oligomeric form. Since no chemicals other than water are added, hydrothermal processes have important advantages over the processes referred above. Nevertheless, as demonstrated by reported data in the literature for other materials, e.g. corn straw (Moniz et al., 2013), corn cobs (Moura et al., 2007), wheat straw (Carvalheiro et al., 2009), brewery's spent grains (Carvalheiro et al., 2004), or eucalypt wood (Garrote and Parajó, 2002), the careful optimisation of the operational conditions is of paramount importance to achieve a high yield and the balanced distribution 